one in my own operated cases, which makes me think that complete transverse tears through the posterior part of the cartilage must be uncommon.

The under-surface of the patella and as much as possible of the opposite semilunar cartilage should be examined as far as the incision allows before closing the joint.

The capsule is sutured with a continuous catgut stitch in two layers. Iodine is again applied to the edges of the wound, which are approximated with a continuous suture of silkworm-gut.

\section{Dressing}

This is a most important part of the treatment of these cases. The tourniquet is still in position and the joint cavity free from blood. A slack dressing will allow effusion when the tourniquet is removed, whilst a very tight bandage will obstruct the circulation and cause oedema below the knee. The best and safest dressing is the following (Bankart):

A generous amount of gauze and wool is applied to the knee-joint whilst the rest of the limb from toes to tourniquet is wrapped in cotton-wool to the thickness of an inch or more. A series of 6-inch bandages is then applied really tightly from below upwards, so that a steady elastic pressure is maintained not only on the kneejoint itself but also upon the area of the limb below and above it. The tourniquet is finally removed and a layer of wool and a firm bandage takes its place. Providing there are no constitutional disturbances this wool compress is left untouched until the stitches are removed on the eighth day, and the patient next day starts walking and begins a course of faradism to his quadriceps. I firmly believe that this simple method of treating operated knees is a most trusty safeguard against the troublesome and indeed dangerous haemarthrosis which occasionally follows other methods.

\section{Post-operative Care}

No splint is necessary and the patient moves his limb as much as he cares to do. Active contraction of the quadriceps muscle with the knee straight may be practised in bed after the second day, and walking, rowing, and cycling exercises are encouraged as soon as the stitches are removed.

\section{Complications}

1. Haematoma occasionally occurs between the skin and the sutured capsule. If the temperature is raised above $100^{\circ} \mathrm{F}$. on the second or third day the dressing should be at once removed and the wound inspected and palpated. If its edges are bluish-red and the operation area is indurated but the joint itself normal a probe must be inserted between two stitches, the blood evacuated, and the wool compress replaced. This generally closes the incident.

2. Haemarthrosis.-The patient is never really comfortable after operation, and his temperature rises with his pain until it may reach $103^{\circ}$ in a day or two. The kneejoint is so distended and painful that morphine may be required. Aspiration at once relieves the patient's pain and the surgeon's anxiety, but gradual subsidence is usual even if nothing is done, though perhaps this inactivity risks infection from the blood stream.

3. Stitch abscess occasionally occurs two or three weeks after the operation, but clears up rapidly on removal or discharge of a small piece of catgut. Usually it is quite superficial and does not delay convalescence.

4. Passive effusion into the knee-joint commonly appears when the patient begins to get up. It is generally attributed to quadriceps insufficiency, and is best treated by daily faradism to the quadriceps with a wool pressure bandage over the joint. The effusion is serous and does no harm if it is actively treated from the outset.

\title{
General Convalescence
}

It is often six or eight weeks before full, free, and painless movement is regained after a straightforward operation for a cartilage injury, but exercise and some forms of sport are possible a good deal earlier, and the patient can usually resume his ordinary life two or three weeks after operation.

The results of these operations are most gratifying to both patient and surgeon, but diagnosis must be exact, and anyone who undertakes knee-joint surgery ought to be able to feel that his technique is as safe as he can make it.

\section{TREATMENT OF SOME ERRORS OF META- BOLISM AT BRITISH SPAS ${ }^{\star}$}

BY

\author{
G. L. KERR PRINGLE, M.C., M.D.Ed. \\ Consulting Physician, Harrogate Royal Bath Hospital
}

After a number of years' practice at a spa one comes to definite conclusions as to the type of case which does best and which obtains the most relief from a "cure" there. My own experience leads me to believe that the most suitable are those due to some error of metabolism in which the liver is the chief offender; among these may be mentioned congestion of the liver, disease of the gallbladder, gout, gouty glycosuria, so-called gouty eczema, some forms of obesity, and certain chronic rheumatic states.

In using the term "spa treatment" I refer to definite balneological treatment-that is, treatment with waters and baths-and not to other forms of physiotherapy, such as diathermy, ultra-violet rays, massage, etc.

During recent years the medical profession would appear to consider that spas are useful only in the treatment of chronic rheumatic diseases, and it is by no means unanimous even on this point, as at the discussion on the treatment of chronic rheumatic diseases at the Oxford meeting Dr. H. L. Tidy (1936) confessed scepticism regarding the advantages of spa treatment over hydrological treatment away from a spa. The profession seems to have forgotten that the spa is primarily and particularly useful in the treatment of disturbances of metabolism.

I had not been long in spa practice before I noticed that patients with disturbances of liver function, simple congestion, enlargement due to long residence in the Tropics or associated with gastro-intestinal catarrh, all did well; cases of plethoric gout and glycosuria associated with gout improved enormously under the administration of mineral waters and baths. Further, it was observed that patients coming for treatment for sciatica or other rheumatic conditions often gave the information that they had been under treatment at a spa a number of years previously for an attack of gall-stones, or gall-bladder disease, from which they had since been quite free; also that certain cases of rheumatism cleared up with waters

* An elaboration of part of a lecture on spa treatment read before the Ayrshire Division of the British Medical Association,
December, 1936. 
alone and without any treatment other than mineral water baths - that is, cases due to metabolic disturbances.

We have in Great Britain a number of spas which are particularly suited for the treatment of these metabolic errors-Cheltenham and Leamington with their sulphated waters, and the sulphur-saline group of Harrogate, Llandrindod, and Strathpeffer. Unfortunately the exact physiological actions of these waters (with the exception of the Old Sulphur Well at Harrogate) have never been worked out, and the evidence of their usefulness is entirely clinical. We know from such clinical evidence that the waters of Cheltenham and Leamington in full doses are purgative in action and stimulate peristalsis; that they are useful in the reduction of fat and abdominal plethora, also in catarrhal jaundice and hepatic congestion, uric acid, gravel, gout, and gouty glycosuria. The sulphursaline waters contain large quantities of sodium chloride, are purgative in action, and effect a marked increase in the output of bile. Laborious investigations on the Old Sulphur Well at Harrogate were carried out first by William Bain and secondly by David Brown and Woodmansey. The physiological and clinical evidence shows that this water is especially useful in abdominal plethora, hepatic congestion, certain forms of toxaemia, gout, sequelae of malaria, metallic poisons such as lead and mercury, some forms of skin disease, and also of rheumatic disease. Harrogate possesses half a dozen other sulphur wells which are used for internal treatment, and which vary in the strength of their chloride and sulphur contents. The waters of Strathpeffer stand in a separate category: the chloride content is small, and the purgative effect is produced by the presence of magnesium salts in fairly large quantities. The sulphur content is mainly in the form of $\mathrm{H}_{2} \mathrm{~S}$.

\section{Congestion of the Liver}

The liver helps to prepare all three food materialsproteins, fats, and carbohydrates-for utilization by the tissues, and acts as a clearing station between the portal and systemic circulation. This much-abused organ is slow to respond to continual insults, and when gastric symptoms reveal dietetic failings, the liver, less obvious in its response, is nevertheless the sufferer. The excessive flow of abnormal intestinal toxins, by placing an unnatural strain on the function of the liver cells, leads to congestion producing tenderness on palpation and a sense of weight in the hypochondrium, and the urine contains large quantities of abnormal constituents.

Congestion of the liver due to overeating, alcoholism, insufficient exercise or stasis in the circulation, sedentary occupation, habitual constipation, or induced by diseases of the heart, lungs, or uterus, improves much by a course of any of these waters. In the Tropics prolonged exposure to high degrees of temperature combined with over-full and over-rich feeding, alcohol, and deficiency of muscular exercise tends to produce a hyperaemia which passes into congestion with blood stasis and sometimes frothy diarrhoea; such patients do remarkably well with any of the above mineral waters.

The benefits derived from the internal use of these waters is materially assisted by the external use of packsmustard and mud-applied over the liver and followed by a needle-bath, along with such hydrological treatment as massage-douches and immersion baths. Early cases of cirrhosis of the liver, even when associated with ascites, do well under such treatment, and improvement is maintained provided there is complete abstinence from alcohol. Where anaemia is associated with these conditions the course of sulphur or sulphated water can be followed up by one of the mild iron waters that are to be found at many of these spas, such as the Kissingen and Crescent saline springs at Harrogate.

\section{Diseases of the Gall-bladder}

In most instances the treatment of this condition is symptomatic, and no attempt is made to strike at the underlying causes. Usually once the case is diagnosed by $x$ rays as one of gall-stones the surgeon is called in. Gall-bladder disease is practically always a secondary condition. Rehfuss and Nelson (1935) consider that there are three fundamental factors in its causation--(1) the metabolic; (2) the problem of infection; (3) the question of stasis.

1. The metabolic factor involves several organs: the liver, which manufactures bile; the gall-bladder itself, which collects the bile and acts as a reservoir and appears also to concentrate the bile, as the bile leaving the gallbladder is more concentrated than the hepatic bile; the nervous mechanism, which concerns the expulsion of bile ; the intestinal canal, which is itself drained by the portal system. Analysis of hepatic and gall-bladder bile shows that the cholesterol content of the latter is much greater than can be accounted for by the concentration which takes place in the gall-bladder. Considerable importance attaches to the bile salts which are elaborated by the liver cell, the cholates which in some way tend to hold cholesterol in solution. The bile acids are divided into two groups-glycocholic acid and taurocholic acid, the former on decomposition yielding glycol and choline, the latter having particular influence on fat metabolism. Fats act as a strong stimulant for the contraction of the gallbladder, and therefore a low fat diet will act as a splint to the gall-bladder by helping to prevent contraction, and a low cholesterol dietary should be beneficial in reducing bile cholesterol. To-day we distinguish choleretic drugs which appear to affect the liver cell, and cholagogues which affect gall-bladder function.

The portal system draining the whole intestinal canal is another metabolic factor of great importance. The majority of gall-bladder cases have a history of constipation with resulting toxaemia and a heavier burden on the liver cell.

2. The Infection Factor.-Most pathologists now accept the view that infection is by the systemic circulation, and it is not probable that it creeps up the common duct from the duodenum. Foci of infection in the teeth, tonsils, sinuses, and bowel, as in arthritis, are presumed to play a large part.

3. Stasis.-This question is still sub judice. German workers at one time laid great stress on this factor. Visceroptosis and the asthenic habitus may have some part in its production.

\section{Treatment of Gall-bladder Cases}

Much will depend on whether the case is one of cholecystitis (i) associated with gall-stones, (ii) without gall-stones, or (iii) jaundice in the non-calculous cholecystitis. Diagnosis of these three varieties is essentiala radiograph is of primary importance, and where there is jaundice the employment of the van den Bergh test is essential. When the condition is associated with the presence of gall-stones the question at once arises whether operation is necessary and when it should be carried out.

Wilkie (1934) recently laid it down that in well-established cases of infection of the biliary passages with gallstones surgery was the rational treatment, but if surgery 
was to be undertaken the preliminary preparation of the patient was as necessary as the operation. For many the operation was a great ordeal; and he referred to the diminished mortality in cholecystectomy due to doubt to increasing surgical experience, but in the main to the increased attention to the pre-operative regime. When cholaemia supervened benefit had been found by the application of heat through the lower part of the chest, and we must get away from the idea that we are not dealing with obstruction due to stone, or irritation due to stone, but with a disordered physiology which affected the liver. If these remarks apply to gall-bladder disease with the presence of calculi, they apply even more to cholecystitis without calculi, and also to non-obstructive jaundice.

Non-surgical treatment is of three kinds: (a) dietetic ; (b) medicinal ; (c) balneological.

(a) Dietetic.-The dietary in the pre-operative case of gall-stones is of great importance. Two points must be observed: (i) to keep the gall-bladder at rest; (ii) to try to diminish the cholesterol content of the bile. Therefore fats and fatty foods should be avoided. Butter, fats, all fried foods, duck, goose, pork, cheese, and fatty fish such as salmon, mackerel, and herring should be removed from the dietary, also eggs as being rich in cholesterol. Milk, as ordinary butter-milk and skimmed milk, may be taken, and vegetables, lean meats, poultry, and non-fatty fish, all either grilled or roasted, but never fried. Where there is no evidence of calculi it may be necessary to assist the gall-bladder to contract, and a diet containing fats may be ordered.

(b) Medicinal.-It has already been shown that there are two varieties of drugs: the choleretic which act on the bile-forming function of the liver, and the cholagogues which act on the gall-bladder function. There is, however, no absolute distinction, because many "cholagogue" drugs have a "choleretic" action. Sodium sulphate is one of the oldest of the cholagogues employed, and is the chief constituent of the springs at Cheltenham, Leamington, and Carlsbad; it has been shown that its administration is followed by a distinct fall in blood cholesterol. There are numerous vegetable cholagogues, such as podophyllin, euonymin, aloes, iridin, etc. Ox bile, however, is probably the best cholagogue known, and the preparations decholin, colalin, or panbiline, prepared from the active constituents of bile, may be employed. Decholin is reputed to have an effect on the liver cells as well as on the gall-bladder. William Bain (1935), working on a human subject with a biliary fistula, showed that the ingestion of the Old Sulphur Spring water at Harrogate promoted an increase in the flow of bile in both total quantity and total solids, and no other drug or mineral water, such as euonymin, iridin, etc., gave such a striking increase. Bain also claimed that this effect was in part due to stimulation of the hepatic cells. Brown and Woodmansey (1929) investigated the effects of this water on the general metabolism; using human subjects, they found that during the period when the water was being taken there was an increase in the amount of total solids excreted by the kidneys and sweat glands. A marked increase in the excretion of sulphur by way of kidneys, bowel, and sweat glands was recorded. This increase was greatly in excess of the amount contained in the water ingested. They also reported an increase in the excretion of total nitrogen, creatinine, uric acid, urea, and hypoxanthin, as well as the elements calcium, potassium, and manganese. The ions of barium and sodium were found to be retained in the body. They therefore conclude that the drinking of strong sulphur water causes an increase in general metabolism. They also found that this water has strong bactericidal properties. Bain made further experiments with the Harrogate springs on the isolated heart and on the intact animal, and found that blood pressure and cardiac systole are both gently increased with the strong sulphur water. These physiological findings support the clinical evidences with which we have been familiar for many years.

(c) Physical and Balneological-At eliminative spas, such as Cheltenham, Leamington, and the sulphur-saline spas, the accompanying constipation may be benefited by intestinal lavage, reinforcing the mineral waters taken by mouth. Heat in the form of mustard or mud packs combined with needle-baths, massage douches, or hot immersion mineral water baths are all aids which are at hand and should not be neglected.

\section{Gout and its Treatment}

Clinical evidence has taught us that certain manifestations of disease, such as occur in eczema, phlebitis, or bronchitis, are of a gouty nature. Gout may be regarded as an inborn error of metabolism, though it is quite possible that foci of infection may be the causal agent in precipitating an attack. Acute gout is not as evident as it was thirty years ago, but there are indications that it is on the increase again, though probably in a less acute form. The young physician of to-day is apt to look on this disease as a pathological rarity, but I am confident that many cases of the milder types are overlooked and are diagnosed as rheumatism, arthritis, or cellulitis. A history of mild attacks in a single joint, the presence of tophi, a history of heredity, biochemical findings-that is, raised blood uric acid over 3.5 per cent.-hypercholesterolaemia, $x$-ray findings-that is, punched-out areas of rarefaction in articular ends of bones- point collectively to a case being one of gout.

The spa treatment of gout comes under two categories -treatment of a recent acute or subacute attack on the one hand, and prophylactic measures against recurrence. In treating acute gout, spa treatment is harmful while any inflammatory signs are still present. This is one of the pitfalls of spa practice, as too energetic treatment is apt to cause a recrudescence of the disease, no matter whether the water of Bath, Buxton, or sulphur spa is employed. A few days' rest in bed on arrival at the spa, with the internal administration of colchicum and calomel, will usually prevent a relapse. The internal administration of mineral waters, such as those of Bath and Buxton and the mild sulphur, can then be employed along with local applications of peat and mud or hot air. Caution must be observed with baths which involve massage or other physical measures, and ordinary exercise must be curtailed. No definite rule can be laid down; the physician has to feel his way with each individual case. Cases of gouty glycosuria usually clear up after three or four days' treatment, and cases of gouty eczema often do so on the sulphur water alone without the aid of alkaline sulphur baths.

Diet is important in all cases. Lockie and Hubbard (1935) have found that diets high in fat and low in carbohydrates and protein tend to precipitate an attack with an increase of hyperuricaemia. These findings have not so far been confirmed in this country, but they should be borne in mind. When spa treatment is used as a prophylactic measure one must still be on one's guard against precipitating an attack, otherwise more active measures may be employed. 
R. G. Gordon (1936) in a recent paper drew attention to the metabolic factor in chronic rheumatism, with special reference to fibrositis, and observed that the pendulum had swung too far, and that we must consider some cases of chronic rheumatism to have a gouty basis, quite apart from the question of the presence of a focus of infection. This same viewpoint $I$ raised at the discussion on chronic rheumatic diseases at the Oxford meeting of the British Medical Association last year. Further, I pointed out that there were cases where there might be no evidence of an infective focus. For instance, patients tell you that they get pains and aches in their muscles (a sprained feeling) after drinking beer or Burgundy ; after eating strawberries, asparagus, etc.; or after taking a purge; and patients suffering from glycosuria often complain of such symptoms. Another group of patients will tell you that when they get out of bed in the morning their feet feel tender, their hands are stiff, and their grip is weak. By the time they have completed dressing this discomfort has passed off. On examination there may be no physical signs beyond perhaps some tenderness on squeezing the metacarpo-phalangeal joints as in a vigorous handshake. Such cases improve much by the use of sulphated or sulphide waters, and by trying to correct the errors of metabolism, quite apart from general physical treatment.

\section{Post-malarial Cachexia}

The waters of these spas are also distinctly useful in the cachexia following malaria. Enlargement of spleen and liver derives much benefit from a course of these waters, combined with baths, exercise, change of air, etc. In the winter and early spring Cheltenham and Leamington are more suitable, while in the summer months the more bracing climate of Harrogate and Strathpeffer works wonders. In fact, I know of no type of case which shows greater and more rapid improvement with a course of these waters. Many residents in the Tropics, from India, Malay, West Coast of Africa, etc., make it a practice to have a "cure" during their periodic leaves home, mainly as a prophylactic measure.

\section{REFERENCES}

Bain, William (1935). The Pharmacological Action of the Harrogate Drinking Waters. Churchill, London.

Brown, D., and Woodmansey, A. (1929). Proc. roy. Soc. Med., $1928-9,22,487$.

Gordon, R. G. (1936). British Medical Journal, 2, 1243.

Lockie, L. M., and Hubbard, R. S. (1935). J. Amer. med. Ass., 104, 2072.

Rehfuss, M. E., and Nelson, G. M. (1935). Medical Treatment of Gall-bladder Disease. Saunders, Philadelphia.

Tidy, H. Letheby (1936). British Medical Journal, 2, 418.

Wilkie, D. P. D. (1934). Ibid., 1, 909.

A comprehensive survey of obesity and emaciation due to pathological causes, by Professor Francesco Galdi of Pisa and three of his colleagues, is published in the proceedings of the forty-second congress of $\mathrm{La}$ Società Italiana di Medicina Interna, held in Rome in October, 1936. The authors comment on the extensive literature, especially from German and American sources, dealing with obesity, and the comparative neglect of pathological loss of flesh. The relation of metabolism, of the nervous system, and of the endocrine glands to these conditions is fully discussed, and some sixty pages are devoted to more detailed separate analyses of the metabolism of proteins, carbohydrates, and fats. Following a description of the pathological anatomy of both conditions, the several forms of obesity and emaciation are considered in their clinical aspects.

\section{POST-SCARLATINAL NEPHRITIS A STUDY IN PREVENTION \\ BY}

\section{B. A. PETERS, M.D.Cantab., D.P.H.}

Medical Superintendent, Ham Green Hospital, Bristol Lecturer in Infectious Diseases, University of Bristol

AND

IRIS M. CULLUM, M.D.Lond.

Late Assistant Resident Medical Officer, Ham Green Hospital, Bristol

By giving large amounts of alkalis in the course of an attack of scarlet fever Carter and Osman (1927) showed that a considerable reduction in the incidence of nephritis occurred in cases so treated compared with a control series. Peters (1932) brought forward evidence showing that the administration of thyroid and iodine for the first fortnight of an attack of scarlet fever was followed by a considerable fall in the incidence of nephritis.

Below is given a table showing the incidence of postscarlatinal nephritis in five-year periods during the past twenty-five years in Ham Green Hospital.

TABLE I

\begin{tabular}{c|c|c|c}
\hline Period & $\begin{array}{c}\text { Total Cases } \\
\text { of } \\
\text { Scarlet Fever }\end{array}$ & $\begin{array}{c}\text { Cases of } \\
\text { Nephritis }\end{array}$ & $\begin{array}{c}\text { Percentage } \\
\text { Incidence } \\
\text { of Nephritis }\end{array}$ \\
\hline $1910-14$ & 2,771 & 53 & 1.92 \\
$1915-19$ & 1,329 & 25 & 1.88 \\
$1920-24$ & 2,496 & 63 & 2.5 \\
$1925-29$ & 3,148 & 28 & 0.88 \\
$1930-34$ & 2,427 & 9 & 0.37 \\
$1935-36$ & 1,350 & 43 & 3.2 \\
\hline
\end{tabular}

Thyroid and iodine were given during the period of 1930-4. Comparing the different incidence in the two periods of lowest prevalence, it will be found that the difference is 2.3 times the standard deviation. The difference is very much higher if the whole twenty-five-year period is taken into consideration. On these grounds we were disposed to think that our method was proving effective, especially as 1930-4 figures include two cases which were admitted with nephritis already present in whom preventive methods had not been applied.

In the winter of 1934-5 a more severe type of scarlet fever appeared. It was characterized by a great increase of secondary attacks whilst in hospital ; by several second attacks in patients within a few weeks of discharge; by the increase of our return case rate to three times its normal number; by an increase of all the more severe complications ; and by the occurrence of attacks of scarlet fever in six Dick-negative reactors among the staff, an event which had not occurred for many years.

In spite of administering thyroid and iodine, cases of nephritis cropped up with distressing frequency. We therefore investigated our method afresh, using alternate cases as test and control. Table II shows the result of this investigation. By the term "albuminuria" is meant symptomless albuminuria for three consecutive days or longer. "Nephritis" includes cases with signs or symptoms of renal failure. The observation of the sedimentation rate of the red blood corpuscles by Cookson (1936), working in this hospital, suggests that there is a sharp line of demarcation between the two conditions. 